\title{
Cognitive Flexibility, Theory of Mind, and Hyperactivity/Inattention
}

\author{
Brad M. Farrant, ${ }^{1,2}$ Janet Fletcher, ${ }^{1}$ and Murray T. Maybery ${ }^{1}$ \\ ${ }^{1}$ School of Psychology (M304), The University of Western Australia, 35 Stirling Highway, Crawley, WA 6009, Australia \\ ${ }^{2}$ Telethon Institute for Child Health Research, The University of Western Australia, P.O. Box 855, West Perth, WA 6872, Australia \\ Correspondence should be addressed to Brad M. Farrant; bfarrant@ichr.uwa.edu.au
}

Received 26 August 2013; Revised 22 November 2013; Accepted 9 December 2013; Published 12 January 2014

Academic Editor: Glenda Andrews

Copyright (c) 2014 Brad M. Farrant et al. This is an open access article distributed under the Creative Commons Attribution License, which permits unrestricted use, distribution, and reproduction in any medium, provided the original work is properly cited.

\begin{abstract}
The present study analyzed the concurrent and longitudinal relations among cognitive flexibility, theory of mind, and hyperactivity/inattention in a sample of 70 typically developing children ( $M$ age $=61.4$ months, $S D=8.3$ months). Mothers and teachers reported on children's hyperactivity/inattention using the strengths and difficulties questionnaire (Goodman, 1997), cognitive flexibility was measured using the dimension change card sort task (Zelazo, 2006), and theory of mind was assessed using a battery of tasks. Cognitive flexibility and theory of mind scores were found to be significantly negatively correlated with the level of hyperactivity/inattention at both time points. Furthermore, year 1 cognitive flexibility score was found to be a significant predictor of year 2 hyperactivity/inattention score after controlling for child age, gender, and year 1 hyperactivity/inattention score. Directions for future research include training studies which would further our understanding of these relationships and allow more effective interventions.
\end{abstract}

\section{Introduction}

The ability to focus attention and regulate behavior is a key determinant of scholastic achievement and occupational success [1-3]. Indeed, these self-control skills are viewed as desirable by parents, teachers, and employers alike [3] and high levels of problems with focusing or sustaining attention and regulating behavior are core clinical features used to diagnose attention-deficit hyperactivity disorder (ADHD). However, many children with symptoms of hyperactivity and inattention do not meet the criteria for a formal ADHD diagnosis and parents and education professionals are confronted with the challenge of working with these children [4]. The present study investigated the cognitive factors associated with symptoms of hyperactivity/inattention in typically developing children during the early school years.

Much of the research into the development of children's self-regulation skills has been conducted within the executive function framework [5]. Inhibitory control, working memory, and cognitive flexibility are components of executive function that contribute to the development of self- regulation [6]. These executive function components follow different trajectories across early development such that the preschool period is characterized by dramatic improvements in inhibitory control, whereas the development of cognitive flexibility accelerates during the early school years [7].

According to cognitive complexity and control (CCC) theory, cognitive flexibility refers to the ability to flexibly shift between multiple incompatible perspectives or descriptions of an object or event [8]. Linguistically formulating conflicting perspectives or descriptions and embedding these under a higher-order rule enable the ability to flexibly shift between these conflicting perspectives or descriptions [810]. This ability to formulate and use higher-order rules enables children to formulate more complex and integrated rule systems which then allow them to control their attention and behavior in a relatively top-down fashion [10, 11]. Perseverative behavior occurs when children lack the ability to integrate incompatible pieces of knowledge into a more complex rule system. Thus, CCC theory predicts that children with more advanced cognitive flexibility skills will have better attention and behavior regulation skills. 
Although we are unaware of any research which directly assesses the relationship between cognitive flexibility and hyperactivity/inattention in typical development there is some research which has investigated the cognitive flexibility skills of children with ADHD. A number of studies have found that cognitive flexibility is impaired in children with ADHD [12-15]. However, other studies have failed to find impaired cognitive flexibility in children with ADHD [16, 17]. A recent meta-analysis also observed inconsistent results regarding whether ADHD is associated with a cognitive flexibility (set-shifting) deficit [18]. One possible explanation for the inconsistency in these findings is that studies of children with ADHD tend to involve children with a wide range of ages. Hence, the role of accelerated cognitive flexibility development in the early school years [7] may be masked in some of these studies by the inclusion of older children. Thus, our understanding of the developmental relationship between cognitive flexibility and hyperactivity/inattention would benefit from research focused on the early school years.

Another issue worthy of consideration is the relationships among cognitive flexibility, theory of mind (ToM), and hyperactivity/inattention. ToM involves the cognitive capacity to impute causal mental states to self and others in order to explain and predict behavior [19]. According to CCC theory, ToM development relies on the development of cognitive flexibility $[9,10,20]$ because the embedding of one set of judgments (or rules) within another allows the ability to flexibly shift between conflicting psychological perspectives [20]. Furthermore, it has been argued that one of the reasons that the development of cognitive flexibility enables better self-control is that "the reasoning involved in inferring mental states is also needed for conceptualizing one's own actions and controlling one's behavior" [20]. In other words, ToM development mediates the relationship between cognitive flexibility and attention and behavior regulation skills.

A number of studies have found a correlation between performance on the dimension change card sort (DCCS) task [21] and performance on ToM tasks in both typically developing children [22-26] and children with autism [27]. The DCCS task measures children's cognitive flexibility by assessing whether they can flexibly shift between two sorting rules (based on color and shape). The findings of longitudinal research with typically developing children using the DCCS and ToM (false belief) tasks also support the argument that cognitive flexibility facilitates ToM development [28].

There is also some evidence supporting the argument that ToM development facilitates the ability to regulate attention and behavior. Several studies have found that ToM is impaired in children and adults with ADHD [29-31]. On the other hand, several studies have failed to find impaired ToM in children with ADHD [32-34]. However, there is also evidence indicating that ToM is impaired in children with attention deficits who do not have a formal diagnosis [35] and a significant negative relationship has been found between ToM scores and attention problems in children clinically referred for attention and behavior problems [36]. Here again the fact that studies of children with ADHD tend to involve children with a wide range of ages provides a possible explanation for the inconsistency in these findings.

In summary, there is some evidence in support of the existence of a relationship between cognitive flexibility and ToM. However, the evidence for relationships between these cognitive factors and hyperactivity/inattention is inconsistent and comes from studies of ADHD rather than typical development. Furthermore, the hypothesized relationships between cognitive flexibility, ToM, and self-regulation discussed above also suggest that ToM may mediate the relationship between cognitive flexibility and hyperactivity/inattention [20]. Thus, our understanding of the cognitive factors associated with symptoms of hyperactivity/inattention in typically developing children would benefit from research designed to test these relationships.

The purpose of the present study was to further our understanding of the developmental relationships among cognitive flexibility, ToM, and hyperactivity/inattention in typical development by examining these relationships concurrently and longitudinally. Thus, cognitive flexibility, ToM, and hyperactivity/inattention were assessed for a group of children at an early point in their schooling (Year 1: Y1) and again one year later (Y2).

Drawing on CCC theory and the findings of previous research, it was hypothesized that more advanced cognitive flexibility and ToM skills would be associated with lower levels of hyperactivity/inattention. It was also predicted that ToM would mediate the concurrent relationship between cognitive flexibility and hyperactivity/inattention. For the longitudinal analyses, it was hypothesized that Y1 cognitive flexibility would predict Y2 ToM after controlling for Y1 ToM and that Y1 cognitive flexibility and ToM would predict Y2 hyperactivity/inattention after controlling for the relevant Y1 scores. We also investigated the possibility that hyperactivity/inattention interferes with the subsequent development of cognitive flexibility and ToM. This was done by testing whether Y1 hyperactivity/inattention predicts Y2 cognitive flexibility and ToM after controlling for the relevant Y1 scores.

\section{Method}

2.1. Participants. The data reported here represent part of a larger research project. All of the Australian children who participated in the present study spoke English as their first language. The participants were children receiving regular education who were recruited via three primary schools spread across working class to upper middle class areas. Complete sets of the relevant data were available for 70 (33 male) children (64 Caucasian, 6 Asian) aged between 47 and 76 months ( $M=61.4$ months, $S D=8.3$ months $)$ at the commencement of the study.

\subsection{Measures}

2.2.1. Dimension Change Card Sort Task. Cognitive flexibility was assessed at Y1 and Y2 using the border version of the dimension change card sort (DCCS) task [21]. The DCCS task requires children to sort test cards that vary on two 
dimensions (object and color) into two trays each of which has a target card attached. The test cards and target cards differ on the dimensional values (test cards: red rabbit and blue boat; target cards: red boat and blue rabbit) so that the test cards can be sorted into the trays by either dimension. In the first (preswitch) phase, children are asked to sort the two types of test cards according to one dimension (e.g., color: red rabbit test card goes with red boat target card and blue boat test card goes with blue rabbit target card). In the second (postswitch) phase, children need to shift and sort according to the other dimension (e.g., shape: red rabbit test card goes with blue rabbit target card and blue boat test card goes with red boat target card). Those children who pass the post-switch phase also complete the third (border) phase which involves alternating between sorting on either of the two dimensions depending on whether the card has a border or not. The children sorted six cards in each of the pre- and post-switch phases and had to correctly sort five or more in order to pass each of these phases and move on to the next phase. Following Zelazo [21], 12 cards were sorted in the border phase and children had to correctly sort 9 or more in order to pass this phase ( $p<.05$, on the basis of the binomial theorem; [37]). As recommended by Zelazo [21], each child's performance was scored as 0 (failed pre-switch phase), 1 (passed pre- but failed post-switch phase), 2 (passed pre- and post-switch but failed the border phase), or 3 (passed all phases).

2.2.2. Theory of Mind. Children's ToM ability was measured using two batteries of tasks, each including one of the versions of Wellman and Liu's [38] ToM scale used by Farrant et al. [39], one of Woolfe et al's [40] low verbal false belief tasks, and one of Sullivan and Tager-Flusberg's [41] second-order false belief tasks (see Appendix for detailed procedure for the ToM tasks). These tasks range in difficulty from the diverse desires task that assesses whether the individual understands that other people can have desires that differ from his/her own through to the second-order false belief task that assesses the ability to attribute second-order or embedded mental states (e.g., he thinks that she thinks). Previous research has found that typically developing children, with few exceptions, pass the Wellman and Liu tasks in a fixed succession between the ages of 45 and 64 months [38, 42]. For the present study, the two versions of Wellman and Liu's [38] ToM scale were modified to include control questions on the diverse desires and diverse beliefs tasks and an own false belief question in the contents false belief task. Thus, each child completed six ToM tasks containing a total of eight target questions (two target questions in each of the first- and second-order false belief tasks). Each ToM target question was scored as 1 (passed) or 0 (failed), and to pass each target question the child also had to answer the relevant memory and/or control questions correctly. This resulted in a maximum possible ToM score of 8 .

2.2.3. Hyperactivity/Inattention. The hyperactivity scale of the strengths and difficulties questionnaire (SDQ; [43]) was completed by mothers and teachers and was used as a measure of hyperactivity/inattention. The hyperactivity scale contains five items that assess the degree to which children display hyperactivity (e.g., restless, overactive, cannot stay still for long) and attention problems (e.g., easily distracted, concentration wanders). Informants rate how well each item describes the child on a scale ranging from (0) not true through (1) somewhat true to (2) certainly true, with a maximum possible score of 10 for the scale. The SDQ is widely used [44] and the hyperactivity scale has been found to have acceptable internal reliability, interrater agreement, and testretest reliability [45]. The reliability of the scale was good for the present sample of mothers at Y1 $(\alpha=.77)$ and Y2 $(\alpha=.80)$ and teachers at Y1 $(\alpha=.91)$ and $\mathrm{Y} 2(\alpha=.90)$.

2.3. Procedure. Consent forms and information sheets were sent to parents/guardians of all kindergarten and preprimary children attending the three mainstream schools. Questionnaires were mailed to the primary caregivers who chose to participate, and completed questionnaires were mailed directly back to the researcher. The strengths and difficulties questionnaire (SDQ; [43]) was completed by primary caregivers and teachers at the beginning of the study (Y1) and again one year later (Y2). In Y1 and Y2, children were individually tested in a quiet room at the child's school. The ToM tasks were completed in one session and the DCCS task was completed in another session. For each year, the maximum period between the first and final session for each participant was three weeks. Each test session lasted approximately 15 minutes. The sessions were completed in a counterbalanced order and the within-session order of presentation of the individual ToM tasks within each task set was also counterbalanced. Approximately half of the children completed one version of the ToM task set in the first year and the other half completed the other version. Each child received the alternate version of the ToM task set in the second year.

\section{Results}

ToM scores were not significantly different between the two task sets at $\mathrm{Y} 1$ or $\mathrm{Y} 2$ (both $t \mathrm{~s}(68)<0.78$, both $p$ s $>$ .44). Therefore, the results were collapsed across the two sets. Descriptive statistics are presented in Table 1. As can be seen in Table 1, children's cognitive flexibility and ToM scores increased significantly between Y1 and Y2 (both Cohen's $d>$ .78), whereas their hyperactivity/inattention scores did not alter significantly (both Cohen's $d<.09$ ). Mothers' and teachers' ratings of children's hyperactivity/inattention were significantly correlated at Y1 and Y2 (both $r s(72)>.43$, both ps < .01). Measures of children's hyperactivity/inattention across different contexts at each time point were created by averaging the $z$ scores for mothers' and teachers' ratings. Multi-informant approaches such as this are preferable as they avoid single-source bias [46] and because different informants provide important information about children's behavior as it varies across different settings [47, 48]. Child gender was coded as zero for male and one for female.

Inspection of the correlations between the measures revealed that the main study variables were associated with 
TABLE 1: Means and standard deviations of scores.

\begin{tabular}{|c|c|c|c|c|c|c|}
\hline \multirow{2}{*}{ Variable } & \multicolumn{2}{|c|}{ Year 1} & \multicolumn{2}{|c|}{ Year 2} & \multicolumn{2}{|c|}{$t$-test } \\
\hline & $M$ & $S D$ & $M$ & $S D$ & $t$ & $p$ \\
\hline Cognitive flexibility (max. score 3 ) & 1.63 & 0.54 & 2.11 & 0.55 & 7.65 & $<.01$ \\
\hline ToM (max. score 8 ) & 4.09 & 1.88 & 5.40 & 1.43 & 6.44 & $<.01$ \\
\hline Mother rated hyperactivity/inattention (max. score 10) & 3.30 & 2.27 & 3.19 & 2.30 & -0.54 & .59 \\
\hline Teacher rated hyperactivity/inattention (max. score 10) & 2.16 & 2.72 & 2.38 & 2.50 & 0.69 & .49 \\
\hline
\end{tabular}

$N=70$.

TABLE 2: Correlations between variables.

\begin{tabular}{|c|c|c|c|c|c|c|c|c|}
\hline Variable & 1 & 2 & 3 & 4 & 5 & 6 & 7 & 8 \\
\hline (1) Y1 cognitive flexibility & - & & & & & & & \\
\hline (2) Y1 ToM & $.52^{\dagger}$ & - & & & & & & \\
\hline (3) Y1 hyperactivity/inattention & $-.42^{\dagger}$ & $-.56^{\dagger}$ & - & & & & & \\
\hline (4) Y2 cognitive flexibility & $.53^{\dagger}$ & $.40^{\dagger}$ & $-.40^{\dagger}$ & - & & & & \\
\hline (5) Y2 ToM & $.48^{\dagger}$ & $.50^{\dagger}$ & $-.38^{\dagger}$ & $.25^{*}$ & - & & & \\
\hline (6) Y2 hyperactivity/inattention & $-.40^{\dagger}$ & $-.35^{\dagger}$ & $.65^{\dagger}$ & $-.41^{\dagger}$ & $-.26^{*}$ & - & & \\
\hline (7) Y1 child age in months & $.36^{\dagger}$ & $.48^{\dagger}$ & $-.29^{\dagger}$ & $.35^{\dagger}$ & $.27^{*}$ & .00 & - & \\
\hline (8) Child gender & $.25^{*}$ & $.24^{*}$ & -.21 & .20 & .19 & -.21 & .03 & - \\
\hline
\end{tabular}

each other in the expected directions and that the degree of multicollinearity between the predictor variables was acceptable (see Table 2). Chronological age and gender were included in the analyses as covariates. Prior to analysis, the data for the study variables were checked for multivariate outliers using Mahalanobis distance with $p<.001$ (critical value $=26.13$ ) but none was found. As hypothesized, both Y1 cognitive flexibility (as measured by the DCCS task) and Y1 ToM were significantly negatively correlated with the hyperactivity/inattention scores at Y1 and Y2 (see Table 2). Similarly, both Y2 cognitive flexibility and Y2 ToM were significantly negatively correlated with Y2 hyperactivity/inattention scores (see Table 2).

3.1. Mediation Analysis. Because of the questionable nature of the assumption that the sampling distributions of the indirect effects are normal and the general superiority of bootstrapping over methods that assume such normality [49], the mediation analysis was conducted using Preacher and Hayes' SPSS multiple mediator macro using 5000 bootstrap resamples [49]. Y1 hyperactivity/inattention (average $z$ score) was entered as the dependent variable, Y1 ToM was entered as the mediator, and Y1 cognitive flexibility was entered as the independent variable. Y1 child age and gender were entered as covariates. This analytic approach provides a stringent test of whether Y1 ToM mediated the effects of Y1 cognitive flexibility on Y1 hyperactivity/inattention. The direct effect of Y1 cognitive flexibility on Y1 hyperactivity/inattention ( $c^{\prime}$ path) was not significant $(b=-.24, p=.17)$. The specific indirect effect via Y1 ToM was significant $(b=-.23$, bias corrected and accelerated $95 \%$ confidence interval -.49 to -.08$)$. Thus, as hypothesized, Y1 ToM mediated the effect of Y1 cognitive flexibility on Y1 hyperactivity/inattention (the mediation analysis was repeated using Y2 hyperactivity/inattention as the dependent variable, Y1 ToM as the mediator, and Y1 cognitive flexibility as the independent variable-this produced a similar pattern of significant results; a further mediation analysis was conducted to test the possibility that cognitive flexibility may mediate the effect of ToM on Y1 hyperactivity/inattention-the results were not significant).

3.2. Longitudinal Analyses. The hypothesized longitudinal relationships among cognitive flexibility, ToM, and hyperactivity/inattention were assessed via a series of hierarchical regressions. In the first hierarchical regression predicting Y2 hyperactivity/inattention (average $z$ score), after controlling for child age, gender, and Y1 hyperactivity/inattention at the first step, as hypothesized, Y1 cognitive flexibility was a significant predictor at the second step (see Regression 1, Table 3). In the second hierarchical regression predicting Y2 hyperactivity/inattention, after controlling for child age, gender, and Y1 hyperactivity/inattention scores at the first step, contrary to the hypothesis, Y1 ToM was not a significant predictor at the second step (see Regression 2, Table 3).

The next analysis found that after controlling for child age, gender, and Y1 ToM at the first step, as hypothesized, Y1 cognitive flexibility significantly predicted Y2 ToM scores (see Regression 1, Table 4). Consistent with the mediation hypothesis Y1 ToM did not significantly predict Y2 cognitive flexibility after controlling for Y1 cognitive flexibility (see Regression 1, Table 5). The final two analyses investigated the possibility that hyperactivity/inattention interferes with the subsequent development of cognitive flexibility and ToM. In the hierarchical regression predicting Y2 ToM, after controlling for child age, gender, and Y1 ToM at the first step, Y1 hyperactivity/inattention was not a significant predictor at the second step (see Regression 2, Table 4). Similarly, in the hierarchical regression predicting Y2 cognitive flexibility, 
TABLE 3: Summary of hierarchical regression analyses for variables predicting year 2 hyperactivity/inattention (average).

\begin{tabular}{|c|c|c|c|c|c|c|}
\hline Variable & $b$ & $\mathrm{SE}(b)$ & $\beta$ & $p$ & Squared part correlation & $\Delta R^{2}$ \\
\hline Step 1 & & & & & & $.47^{*}$ \\
\hline Y1 child age in months & .02 & .01 & .21 & .03 & .04 & \\
\hline Child gender & -.12 & .16 & -.07 & .47 & .00 & \\
\hline Y1 hyperactivity/inattention & .80 & .11 & .70 & $<.01$ & .43 & \\
\hline \multicolumn{7}{|l|}{ Regression 1} \\
\hline Step 2 & & & & & & $.04^{*}$ \\
\hline Y1 cognitive flexibility & -.37 & .16 & -.22 & .03 & .04 & \\
\hline \multicolumn{7}{|l|}{ Regression 2} \\
\hline Step 2 & & & & & & .00 \\
\hline Y1 ToM & -.04 & .06 & -.08 & .49 & .00 & \\
\hline
\end{tabular}

TABLE 4: Summary of hierarchical regression analyses for variables predicting year 2 ToM.

\begin{tabular}{|c|c|c|c|c|c|c|}
\hline Variable & $b$ & $\mathrm{SE}(b)$ & $\beta$ & $p$ & Squared part correlation & $\Delta R^{2}$ \\
\hline Step 1 & & & & & & $.25^{*}$ \\
\hline Y1 child age in months & .01 & .02 & .05 & .70 & .00 & \\
\hline Child gender & .21 & .31 & .07 & .51 & .01 & \\
\hline Y1 ToM & .35 & .10 & .45 & $<.01$ & .15 & \\
\hline \multicolumn{7}{|l|}{ Regression 1} \\
\hline Step 2 & & & & & & $.06^{*}$ \\
\hline Y1 cognitive flexibility & .77 & .32 & .29 & .02 & .06 & \\
\hline \multicolumn{7}{|l|}{ Regression 2} \\
\hline Step 2 & & & & & & .01 \\
\hline Y1 hyperactivity/inattention & -.26 & .23 & -.15 & .26 & .01 & \\
\hline
\end{tabular}

TABLE 5: Summary of hierarchical regression analyses for variables predicting year 2 cognitive flexibility.

\begin{tabular}{|c|c|c|c|c|c|c|}
\hline Variable & $b$ & SE $(b)$ & $\beta$ & $p$ & Squared part correlation & $\Delta R^{2}$ \\
\hline Step 1 & & & & & & $.32^{*}$ \\
\hline Y1 child age in months & .01 & .01 & .19 & .09 & .03 & \\
\hline Child gender & .09 & .12 & .08 & .45 & .01 & \\
\hline Y1 cognitive flexibility & .45 & .12 & .44 & $<.01$ & .16 & \\
\hline \multicolumn{7}{|l|}{ Regression 1} \\
\hline Step 2 & & & & & & .01 \\
\hline Y1 ToM & .03 & .04 & .10 & .47 & .01 & \\
\hline \multicolumn{7}{|l|}{ Regression 2} \\
\hline Step 2 & & & & & & .03 \\
\hline Y1 hyperactivity/inattention & -.13 & .08 & -.18 & .12 & .03 & \\
\hline
\end{tabular}

${ }^{*} p<.05, N=70$.

after controlling for child age, gender, and Y1 cognitive flexibility at the first step, Y1 hyperactivity/inattention was not a significant predictor at the second step (see Regression 2, Table 5).

\section{Discussion}

The present study sought to further investigate the developmental relationships among cognitive flexibility, ToM, and hyperactivity/inattention. This was achieved by examining these relationships concurrently and longitudinally in a sample of typically developing children. Cognitive flexibility, ToM, and hyperactivity/inattention were assessed at an early point in schooling (Y1) and again one year later (Y2).

4.1. Concurrent and Longitudinal Relationships among Cognitive Flexibility, ToM, and Hyperactivity/Inattention. As hypothesized, more advanced cognitive flexibility and ToM skills were associated with lower concurrent levels of hyperactivity/inattention. Furthermore, as predicted, Y1 ToM 
mediated the relationship between Y1 cognitive flexibility and Y1 hyperactivity/inattention. The mediation hypothesis was also supported by the finding that $\mathrm{Y} 1$ cognitive flexibility significantly predicted $\mathrm{Y} 2 \mathrm{ToM}$ scores after controlling for Y1 ToM, whereas Y1 ToM did not significantly predict Y2 cognitive flexibility after controlling for Y1 cognitive flexibility. Thus the current findings provide some evidence that ToM development may mediate the relationship between cognitive flexibility and attention and behavior regulation skills. A possible explanation for this is that the development of cognitive flexibility enables better self-control because "the reasoning involved in inferring mental states is also needed for conceptualizing one's own actions and controlling one's behavior" [20]. However, Y1 cognitive flexibility, but not Y1 ToM, significantly predicted Y2 hyperactivity/inattention after controlling for child age, gender, and Y1 hyperactivity/inattention.

Combining Hughes and Ensor's [50] argument with the findings of Hughes' [7] review may help to explain these results. Hughes and Ensor [50] argued that ToM skills are particularly important for behavior development in the preschool period, whereas executive functioning is more important for subsequent development and Hughes [7] found that the preschool period is characterized by dramatic improvements in inhibitory control, whereas the development of cognitive flexibility accelerates during the early school years. Thus, it may be that advances in ToM and inhibitory control are important for behavior development in the pre-school, period whereas advances in cognitive flexibility are more important for subsequent development during the early school years. However, it could also be that the integration of the executive function component processes of inhibitory control, working memory, and cognitive flexibility [51] contributes to children's performance on the DCCS and ToM tasks used in the present study and that this integration is inversely related to the development of hyperactive behavior. Further research is required to differentiate between these alternative explanations.

Nevertheless, the current findings are consistent with the argument that the development of cognitive flexibility enables better self-control [20] and the findings of previous research that support a developmental relationship between cognitive flexibility and hyperactivity/inattention [12-15]. The current results are also consistent with the finding that training on a card sort task improved children's performance on the task as well as their ability to inhibit behavioral responses on another task [52]. This indicates that practicing shifting between multiple conflicting perspectives or descriptions enhances children's ability to do so as well as their ability to regulate their behavior more generally. This kind of convergence between the findings of correlational analyses of realworld relationships and the results of training studies also allows more certainty regarding causal inferences because the particular strengths of each type of research compensate for the weaknesses of the other $[53,54]$. Furthermore, in the current study, there was no evidence to support causation in the opposite direction (Y1 hyperactivity/inattention did not significantly predict Y2 cognitive flexibility or ToM).
4.2. Implications, Strengths and Limitations, and Directions for Future Research. Combining the results of the current study with the findings of previous research indicating that cognitive flexibility training facilitates behavior regulation [52] suggests that an intervention focusing on improving cognitive flexibility may prove effective in addressing hyperactivity/inattention problems. Cognitive flexibility training research of this kind could also investigate whether, as suggested by Jacques and Zelazo [9], helping children who linguistically label the relevant dimension(s) of an object or event scaffolds their performance until such time as they learn to do this spontaneously.

The use of a multi-informant design is one of the strengths of the current study. However, the fact that mother and teacher ratings of children's hyperactivity/inattention did not change significantly between Y1 and Y2 in the present study may be problematic in that children's behavior may have been rated in comparison to other children of the same age rather than in some absolute sense.

There is empirical and clinical evidence which indicates that hyperactivity and inattention can represent separate dimensions with different outcomes [55, 56]. This opens up a range of alternative scenarios such as the possibility that inattention problems could result in delayed ToM development. Unfortunately, it was not possible to test this hypothesis in the current study because previous research has shown that the hyperactivity scale from the strengths and difficulties questionnaire is not capable of differentiating between attention deficits and hyperactivity/impulsivity [57]. This also meant that it was not possible to assess whether cognitive flexibility and/or ToM development have different effects on hyperactivity versus inattention. Therefore, future research using scales that provide separate measures hyperactivity and inattention such as the Conners [58] and/or observational measures of hyperactivity and inattention should further investigate the relationships found in the current study. Our understanding of the relationships among cognitive flexibility, ToM, and hyperactivity and inattention problems would also benefit from longitudinal research that measures these constructs plus inhibitory control and working memory at regular intervals from a point early in childhood. Such research should also include measures of verbal and nonverbal intelligence.

\section{Conclusions}

The results of the current study indicate that children with more advanced cognitive flexibility skills demonstrate lower levels of hyperactivity/inattention. The findings provide some support for the argument that the development of cognitive flexibility enables better self-control [20] and are the first to suggest that ToM development may mediate the negative relationship between cognitive flexibility and hyperactivity/inattention in early development. Perhaps most importantly, given the scale of hyperactivity/inattention problems in childhood, the present findings add to the empirical evidence which suggests that cognitive flexibility training may provide an effective way to address these problems. 


\section{Appendix}

\section{Detailed Procedure for ToM Tasks}

Diverse Desires. Wellman and Liu's [38] diverse desires task assesses whether the individual understands that other people can have desires that differ from his/her own. The child is shown a doll and a page depicting a carrot and a cookie. "Here's Mr. Jones. It's snack time, so, Mr. Jones wants a snack to eat. Here are two different snacks: a carrot and a cookie." The child is asked the own-desire question:

"Which snack would you like best? Would you like a carrot or a cookie best?"

If the child chooses the cookie (carrot): "Well, that's a good choice, but Mr. Jones really likes carrots (cookies). He doesn't like cookies (carrots). What he likes best are carrots (cookies)." The child is asked the target question:

"So now it's time to eat. Mr. Jones can only choose one snack, just one. Which snack will Mr. Jones choose? A carrot or a cookie?"

The child is asked the control question:

"Which snack does Mr Jones like best? Carrots or cookies?"

The second (analogue) version of the diverse desires task was identical to the first except that the child was told it was playtime and that "Teddy" the teddy bear wanted a toy to play with and could choose between a doll and a truck.

Diverse Beliefs. Wellman and Liu's [38] diverse beliefs task assesses whether the individual understands that other people can have beliefs that differ from his/her own. The child is shown a doll and a page depicting bushes and a shed. "Here's Mr. Jones. Mr. Jones wants to find his cat. His cat might be hiding in the bushes or in the shed." The child is asked the own-belief question:

"Where do you think the cat is? In the bushes or in the shed?"

If the child chooses the bushes (shed): "Well, that's a good idea, but Mr. Jones thinks his cat is in the shed (bushes). He thinks his cat is in the shed (bushes)." The child is asked the target question:

"So where will Mr. Jones look for his cat? In the bushes or the shed?"

The child is asked the control question:

"Where does Mr. Jones thinks his cat is? In the bushes or the shed?"

The procedure for the second diverse beliefs task was identical to the first except that the child was told that "Teddy" was looking for the jam which might be in the cupboard or in the fridge.

Knowledge Access. Wellman and Liu's [38] knowledge access task assesses if the individual understands that whether or not someone knows something depends on whether they have had access to the relevant information. The child is shown a nondescript opaque plastic box with a closed drawer containing a small plastic toy dog inside. "Here's a drawer. What do you think is inside the drawer?" (the child's answer is immaterial.) The drawer is opened and the child is shown the content of the drawer: "Let's see ... it's really a dog inside!" The drawer is closed and the child is asked the control question:

"Okay, what is in the drawer?"

A doll is produced: "Mr. Jones has never seen inside this drawer. Now here comes Mr. Jones." The child is asked the target question:

"So, does Mr. Jones know what is in the drawer?"

Followed by the memory question:

"Did Mr. Jones see inside this drawer?"

To be scored correct, the child must answer "dog" to the control question and "no" to both the target and memory questions. The procedure for the second knowledge access task was identical to the first except that the props were the teddy bear and a nondescript case containing a cracker biscuit.

Contents False Belief. Wellman and Liu's [38] contents false belief task assesses whether the individual understands that people can hold false beliefs about reality. The child is shown a clearly identifiable closed Band-Aid box with a small plastic toy pig inside. "Here's a Band-Aid box. What do you think is inside the Band-Aid box?" "Let's see ... it's really a pig inside!" The Band-Aid box is closed and the child is asked the control question:

"Okay, what is in the Band-Aid box?"

A doll is produced: "Mr. Jones has never seen inside this Band-Aid box. Now here comes Mr. Jones." Then the child is asked the first target question (others false belief):

"So what does Mr. Jones think is in the box? Band-Aids or a pig?"

Followed by the memory question:

"Did Mr. Jones see inside this box?"

Then the second target question (own false belief):

"What did you first think was inside the BandAids box before we opened it? Band-Aids or a pig?"

The procedure for the second contents false belief task was identical to the first except that the props were the teddy bear and a Smarties box with crayons inside.

Low Verbal False Belief. To check the child's understanding of "thought bubbles," the child is shown two pictures, one depicting a girl thinking about a bottle (a girl with an attached thought bubble containing a bottle) and the other depicting 
a girl holding a bottle and is asked "Which girl is thinking about a bottle?" The child is shown a picture of a boy holding a fishing rod with the line going into the water and a flap (which depicts a plausible obstruction (reeds)) that covers what is on the end of the line (a boot). "See this water? Look. It has fish in it. This is Fred. Fred thinks he has caught a fish on the fishingline. Can you cover Fred with your hand while we have a look at what is really on the fishing-line?" This emphasizes Fred's ignorance of what is on the fishing-line. After the flap is replaced, the child is shown a separate picture of Fred with a blank thought bubble above his head. Next to this picture are four small pictures. Two of these pictures are of distracter items, one shows the content of Fred's belief (a fish) and the other shows the actual object (a boot). Then the child is asked the target question:

"Can you point to what Fred thinks is on the fishing-line?"

Then it is followed by the control question:

"Can you point to what is really on the fishingline?"

To be scored correct, the child must point to the fish in response to the target question and to the boot in response to the control question. The procedure for the second low verbal false belief task was identical to the first except that the protagonist Charlie thinks there is a fish under the reeds when it is really a mermaid.

Second-Order False Belief. The child is shown a picture of a boy and his mum. "This is Andrew, and this is his Mum. Tonight it's Andrew's birthday, and Mum is surprising him with a kitten. Mum wants it to be a secret, so she has hidden the kitten in the garden shed. Andrew says, "Mum, I really hope you get me a kitten for my birthday." Remember, Mum wants to surprise Andrew with a kitten. So, instead of telling Andrew she got him a kitten, Mum says, "Sorry Andrew, I did not get you a kitten for your birthday. I got you a really great toy instead."' The child is then asked the first-order false belief target question:

"So what does Andrew think his Mum got him for his birthday? A kitten or a toy?"

(If correct: That's right, Andrew thinks he's getting a toy; if incorrect: But remember, Andrew thinks he's getting a toy) Then it is followed by the first memory control question:

"What did Mum really get Andrew for his birthday? A kitten or a toy?"

(If correct: That's right, Mum wants to surprise Andrew with a kitten; if incorrect: But remember, Mum wants to surprise Andrew with a kitten).

"Now, Andrew says to Mum, "I'm going outside to play." On his way outside, Andrew decides to get his roller-skates from the garden shed. When he opens the door to the garden shed, Andrew finds the birthday kitten! (child is shown a picture of a kitten in a shed) Andrew says to himself, "wow, Mum didn't get me a toy, she really got me a kitten for my birthday." Now, the important thing is that Mum did not see Andrew go to the garden shed and find the birthday kitten." The child is then asked the second memory control question:

"Does Andrew know that his Mum got him a kitten for his birthday?"

(If yes: That's right, Andrew saw the kitten in the garden shed; if no: But remember, Andrew saw the kitten in the garden shed) Then it is followed by the third memory control question:

"Does Mum know that Andrew saw the birthday kitten in the garden shed?"

(If no: That's right, Mum did not see Andrew go to the garden shed and find the kitten; if yes: But remember, Mum did not see Andrew go to the garden shed and find the kitten).

"While Andrew is outside, Andrew's grandmother drops in for a chat with Andrew's mum. (Child is shown a picture of mum and grandma) Grandma asks Mum, "Does Andrew know what you really got him for his birthday?"' The child is then asked the second-order ignorance question:

\section{"What does Mum say to Grandma?"}

"Now remember, Mum does not know that Andrew saw what she got him for his birthday. Then, Grandma asks Mum, "What does Andrew think you got him for his birthday?"” The child is then asked the second order false belief target question:

\section{"What does Mum say to Grandma?"}

It is followed by the justification question:

"Why does Mum say that?"

To be scored correct on the first-order false belief, the child must answer both the first-order false belief target question and the first memory control question correctly. To be scored correct on the second-order false belief, the child must answer all of the questions correctly. The procedure for the other second-order false belief task was identical to the first except that the child (Molly) wants a bike for her birthday but her dad tells her he got her a really great toy instead.

\section{Conflict of Interests}

The authors declare that there is no conflict of interests regarding the publication of this paper.

\section{Acknowledgments}

The authors wish to thank all of the children, families, and teachers for their generous support and participation in this study. They wish to particularly acknowledge the assistance received from the participants' teachers (Fleur Aris, Susan Bamblett, Chris Blamey, Emily Bradshaw, Sarah Bye, Karen Colkin, Julie Cummings, Martine Damon, Pamela Dow, Lesley Edelman, Julie Excell, Linda Goss, Michelle Green, Terri Hale, Martija Jukic, Jill Mitchell, Suzie Morrison, 
Christine Onley, Judith Rangihaeata, Deb Scarterfield, Jenny Smith, Lucy Stewart, Christine Tarnowy, Carolyn Vuletic, Jenny Whiting, Michelle Willis, Megan Wilson, and Veni Zeid).

\section{References}

[1] S. Normandeau and F. Guay, "Preschool behavior and firstgrade school achievement: the mediational role of cognitive self-control," Journal of Educational Psychology, vol. 90, no. 1, pp. 111-121, 1998.

[2] L. S. Fuchs, D. Fuchs, D. L. Compton et al., "The cognitive correlates of third-grade skill in arithmetic, algorithmic computation, and arithmetic word problems," Journal of Educational Psychology, vol. 98, no. 1, pp. 29-43, 2006.

[3] G. Farkas, "Cognitive skills and noncognitive traits and behaviors in stratification processes," Annual Review of Sociology, vol. 29, pp. 541-562, 2003.

[4] M. K. Demaray and L. N. Jenkins, "Relations among academic enablers and academic achievement in children with and without high levels of parent-rated symptoms of inattention, impulsivity, and hyperactivity," Psychology in the Schools, vol. 48, no. 6, pp. 573-586, 2011.

[5] Q. Zhou, S. H. Chen, and A. Main, "Commonalities and differences in the research on children's effortful control and executive function: a call for an integrated model of selfregulation," Child Development Perspectives, vol. 6, no. 2, pp. 112-121, 2012.

[6] M. M. McClelland and C. E. Cameron, "Self-regulation in early childhood: improving conceptual clarity and developing ecologically valid measures," Child Development Perspectives, vol. 6, no. 2, pp. 136-142, 2012.

[7] C. Hughes, "Changes and challenges in 20 years of research into the development of executive functions," Infant and Child Development, vol. 20, no. 3, pp. 251-271, 2011.

[8] S. Jacques and P. D. Zelazo, "On the possible roots of cognitive flexibility," in The Development of Social Cognition and Communication, pp. 53-81, Lawrence Erlbaum Associates Publishers, Mahwah, NJ, USA, 2005.

[9] S. Jacques and P. D. Zelazo, "Language and the development of cognitive flexibility: implications for theory of mind," in Why Language Matters for Theory of Mind, J. W. Astington and J. A. Baird, Eds., pp. 144-162, Oxford University Press, New York, NY, USA, 2005.

[10] P. D. Zelazo, U. Müller, D. Frye et al., "The development of executive function: cognitive complexity and control-revised," Monographs of the Society for Research in Child Development, vol. 68, no. 3, pp. 93-119, 2003.

[11] K. Happaney and P. D. Zelazo, "Inhibition as a problem in the psychology of behavior," Developmental Science, vol. 6, no. 5, pp. 468-470, 2003.

[12] G. M. Marzocchi, J. Oosterlaan, A. Zuddas et al., "Contrasting deficits on executive functions between ADHD and reading disabled children," Journal of Child Psychology and Psychiatry and Allied Disciplines, vol. 49, no. 5, pp. 543-552, 2008.

[13] M. J. Reader, E. L. Harris, L. J. Schuerholz, and M. B. Denckla, "Attention deficit hyperactivity disorder and executive dysfunction," Developmental Neuropsychology, vol. 10, no. 4, pp. 493$512,1994$.

[14] J. Holmes, S. E. Gathercole, M. Place, T. P. Alloway, J. G. Elliott, and K. A. Hilton, "The diagnostic utility of executive function assessments in the identification of ADHD in Children," Child and Adolescent Mental Health, vol. 15, no. 1, pp. 37-43, 2010.

[15] M. Martel, M. Nikolas, and J. T. Nigg, "Executive function in adolescents with ADHD," Journal of the American Academy of Child and Adolescent Psychiatry, vol. 46, no. 11, pp. 1437-1444, 2007.

[16] H. M. Geurts, S. Verté, J. Oosterlaan, H. Roeyers, and J. A. Sergeant, "How specific are executive functioning deficits in attention deficit hyperactivity disorders and autism?" Journal of Child Psychology and Psychiatry and Allied Disciplines, vol. 45, no. 4, pp. 836-854, 2004.

[17] B. A. Corbett, L. J. Constantine, R. Hendren, D. Rocke, and S. Ozonoff, "Examining executive functioning in children with autism spectrum disorder, attention deficit hyperactivity disorder and typical development," Psychiatry Research, vol. 166, no. 2-3, pp. 210-222, 2009.

[18] E. G. Willcutt, A. E. Doyle, J. T. Nigg, S. V. Faraone, and B. F. Pennington, "Validity of the executive function theory of attention-deficit/ hyperactivity disorder: a meta-analytic review," Biological Psychiatry, vol. 57, no. 11, pp. 1336-1346, 2005.

[19] D. Premack and G. Woodruff, "Does the chimpanzee have a theory of mind?" Behavioral and Brain Sciences, vol. 1, no. 4, pp. 515-526, 1978.

[20] D. Frye, P. D. Zelazo, and J. A. Burack, "Cognitive complexity and control. I: theory of mind in typical and atypical development," Current Directions in Psychological Science, vol. 7, no. 4, pp. 116-121, 1998.

[21] P. D. Zelazo, "The Dimensional Change Card Sort (DCCS): a method of assessing executive function in children," Nature Protocols, vol. 1, no. 1, pp. 297-301, 2006.

[22] D. Frye, P. D. Zelazo, and T. Palfai, "Theory of mind and rulebased reasoning," Cognitive Development, vol. 10, no. 4, pp. 483527, 1995.

[23] B. Lang and J. Perner, "Understanding of intention and false belief and the development of self-control," British Journal of Developmental Psychology, vol. 20, no. 1, pp. 67-76, 2002.

[24] U. Müller, P. D. Zelazo, and S. Imrisek, "Executive function and children's understanding of false belief: how specific is the relation?" Cognitive Development, vol. 20, no. 2, pp. 173-189, 2005.

[25] J. Perner, B. Lang, and D. Kloo, "Theory of mind and selfcontrol: more than a common problem of inhibition," Child Development, vol. 73, no. 3, pp. 752-767, 2002.

[26] G. Andrews, G. S. Halford, K. M. Bunch, D. Bowden, and T. Jones, "Theory of mind and relational complexity," Child Development, vol. 74, no. 5, pp. 1476-1499, 2003.

[27] E. Colvert, D. Custance, and J. Swettenham, "Rule-based reasoning and theory of mind in autism: a commentary on the work of Zelazo, Jacques, Burack and Frye," Infant and Child Development, vol. 11, no. 2, pp. 197-200, 2002.

[28] B. M. Farrant, M. T. Maybery, and J. Fletcher, "Language, cognitive flexibility, and explicit false belief understanding: longitudinal lnalysis in typical development and specific language impairment," Child Development, vol. 83, no. 1, pp. 223-235, 2012.

[29] A. Nydén, L. Niklasson, O. Stahlberg et al., "Adults with autism spectrum disorders and ADHD neuropsychological aspects," Research in Developmental Disabilities, vol. 31, no. 6, pp. 1659$1668,2010$.

[30] J. K. Buitelaar, M. van der Wees, H. Swaab-Barneveld, and R. J. van der Gaag, "Theory of mind and emotion-recognition functioning in autistic spectrum disorders and in psychiatric control 
and normal children," Development and Psychopathology, vol.11, no. 1, pp. 39-58, 1999.

[31] J. K. Buitelaar, H. Swaab, M. van der Wees, M. Wildschut, and R. J. van der Gaag, "Neuropsychological impairments and deficits in theory of mind and emotion recognition in a non-autistic boy," European Child and Adolescent Psychiatry, vol. 5, no. 1, pp. 44-51, 1996.

[32] J. Perner, W. Kain, and P. Barchfeld, "Executive control and higher-order theory of mind in children at risk of ADHD," Infant and Child Development, vol. 11, no. 2, pp. 141-158, 2002.

[33] T. Charman, F. Carroll, and C. Sturge, "Theory of mind, executive function and social competence in boys with ADHD," Emotional and Behavioural Difficulties, vol. 6, no. 1, pp. 31-49, 2001.

[34] M. J. Dyck, K. Ferguson, and I. M. Shochet, "Do autism spectrum disorders differ from each other and from nonspectrum disorders on emotion recognition tests?" European Child and Adolescent Psychiatry, vol. 10, no. 2, pp. 105-116, 2001.

[35] T. C. Papadopoulos, G. Panayiotou, G. Spanoudis, and D. Natsopoulos, "Evidence of poor planning in children with attention deficits," Journal of Abnormal Child Psychology, vol. 33, no. 5, pp. 611-623, 2005.

[36] C. M. Fahie and D. K. Symons, "Executive functioning and theory of mind in children clinically referred for attention and behavior problems," Journal of Applied Developmental Psychology, vol. 24, no. 1, pp. 51-73, 2003.

[37] D. Hongwanishkul, K. R. Happaney, W. S. C. Lee, and P. D. Zelazo, "Assessment of hot and cool executive function in young children: age-related changes and individual differences," Developmental Neuropsychology, vol. 28, no. 2, pp. 617-644, 2005.

[38] H. M. Wellman and D. Liu, "Scaling of theory-of-mind tasks," Child Development, vol. 75, no. 2, pp. 523-541, 2004.

[39] B. M. Farrant, J. Fletcher, and M. T. Maybery, "Specific language impairment, theory of mind, and visual perspective taking: evidence for simulation theory and the developmental role of language," Child Development, vol. 77, no. 6, pp. 1842-1853, 2006.

[40] T. Woolfe, S. C. Want, and M. Siegal, "Signposts to development: theory of mind in deaf children," Child Development, vol. 73, no. 3, pp. 768-778, 2002.

[41] K. Sullivan and H. Tager-Flusberg, "Second-order belief attribution in Williams syndrome: intact or impaired?" American Journal on Mental Retardation, vol. 104, no. 6, pp. 523-532, 1999.

[42] C. C. Peterson, H. M. Wellman, and D. Liu, "Steps in theory-ofmind development for children with deafness or autism," Child Development, vol. 76, no. 2, pp. 502-517, 2005.

[43] R. Goodman, "The strengths and difficulties questionnaire: a research note," Journal of Child Psychology and Psychiatry, vol. 38, no. 5, pp. 581-586, 1997.

[44] A. Goodman and R. Goodman, "Population mean scores predict child mental disorder rates: validating SDQ prevalence estimators in Britain," Journal of Child Psychology and Psychiatry and Allied Disciplines, vol. 52, no. 1, pp. 100-108, 2011.

[45] R. Goodman, "Psychometric properties of the strengths and difficulties questionnaire," Journal of the American Academy of Child and Adolescent Psychiatry, vol. 40, no. 11, pp. 1337-1345, 2001.

[46] T. Lewis, J. Kotch, R. Thompson et al., "Witnessed violence and youth behavior problems: a multi-informant study," American Journal of Orthopsychiatry, vol. 80, no. 4, pp. 443-450, 2010.
[47] E. Youngstrom, R. Loeber, and M. Stouthamer-Loeber, "Patterns and correlates of agreement between parent, teacher, and male adolescent ratings of externalizing and internalizing problems," Journal of Consulting and Clinical Psychology, vol. 68, no. 6, pp. 1038-1050, 2000.

[48] J. M. Nelson and H. R. Harwood, "A meta-analysis of parent and teacher reports of depression among students with learning disabilities: evidence for the importance of multi-informant assessment," Psychology in the Schools, vol. 48, no. 4, pp. 371384, 2011.

[49] K. J. Preacher and A. F. Hayes, "Asymptotic and resampling strategies for assessing and comparing indirect effects in multiple mediator models," Behavior Research Methods, vol. 40, no. 3, pp. 879-891, 2008.

[50] C. Hughes and R. Ensor, "Does executive function matter for preschoolers' problem behaviors?" Journal of Abnormal Child Psychology, vol. 36, no. 1, pp. 1-14, 2008.

[51] N. Garon, S. E. Bryson, and I. M. Smith, "Executive function in preschoolers: a review using an integrative framework," Psychological Bulletin, vol. 134, no. 1, pp. 31-60, 2008.

[52] S. M. Dowsett and D. J. Livesey, “The development of inhibitory control in preschool children: effects of "executive skills" training," Developmental Psychobiology, vol. 36, no. 2, pp. 161-174, 2000.

[53] L. Bradley and P. Bryant, "Categorizing sounds and learning to read: a causal connection," Nature, vol. 301, no. 5899, pp. 419421, 1983.

[54] P. L. Harris, "Conversation, pretense, and theory of mind," in Why Language Matters for Theory of Mind, J. W. Astington and J. A. Baird, Eds., pp. 70-83, Oxford University Press, New York, NY, USA, 2005.

[55] A. Mazaheri, C. Fassbender, S. Coffey-Corina et al., "Differential oscillatory electroencephalogram between attentiondeficit/hyperactivity disorder subtypes and typically developing adolescents," Biological Psychiatry, 2013.

[56] J. Pingault, S. Cote, C. Galera et al., "Childhood trajectories of inattention, hyperactivity and oppositional behaviors and prediction of substance abuse/dependence: a 15-year longitudinal population-based study," Molecular Psychiatry, vol. 18, no. 7, pp. 806-812, 2013.

[57] A. K. Ullebø, M.-B. Posserud, E. Heiervang, C. Gillberg, and C. Obel, "Screening for the attention deficit hyperactivity disorder phenotype using the strength and difficulties questionnaire," European Child and Adolescent Psychiatry, vol. 20, no. 9, pp. 451458, 2011.

[58] C. K. Conners, Conners Comprehensive Behavior Rating Scale Manual, Multi-Health Systems, Ontario, Canada, 2008. 

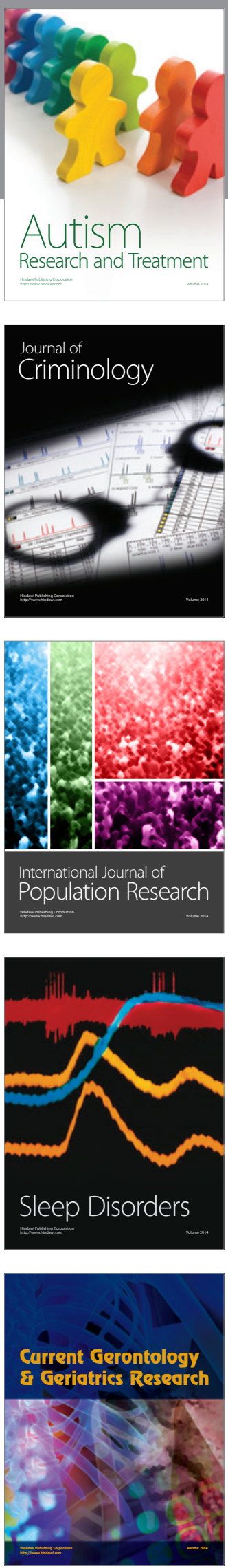
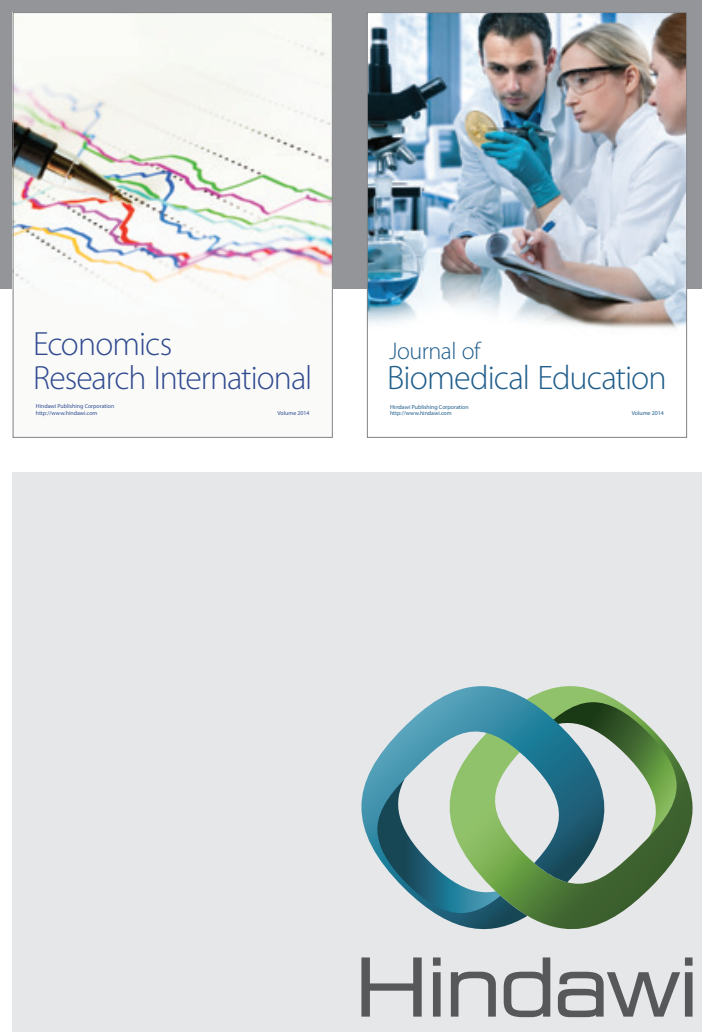

Submit your manuscripts at

http://www.hindawi.com

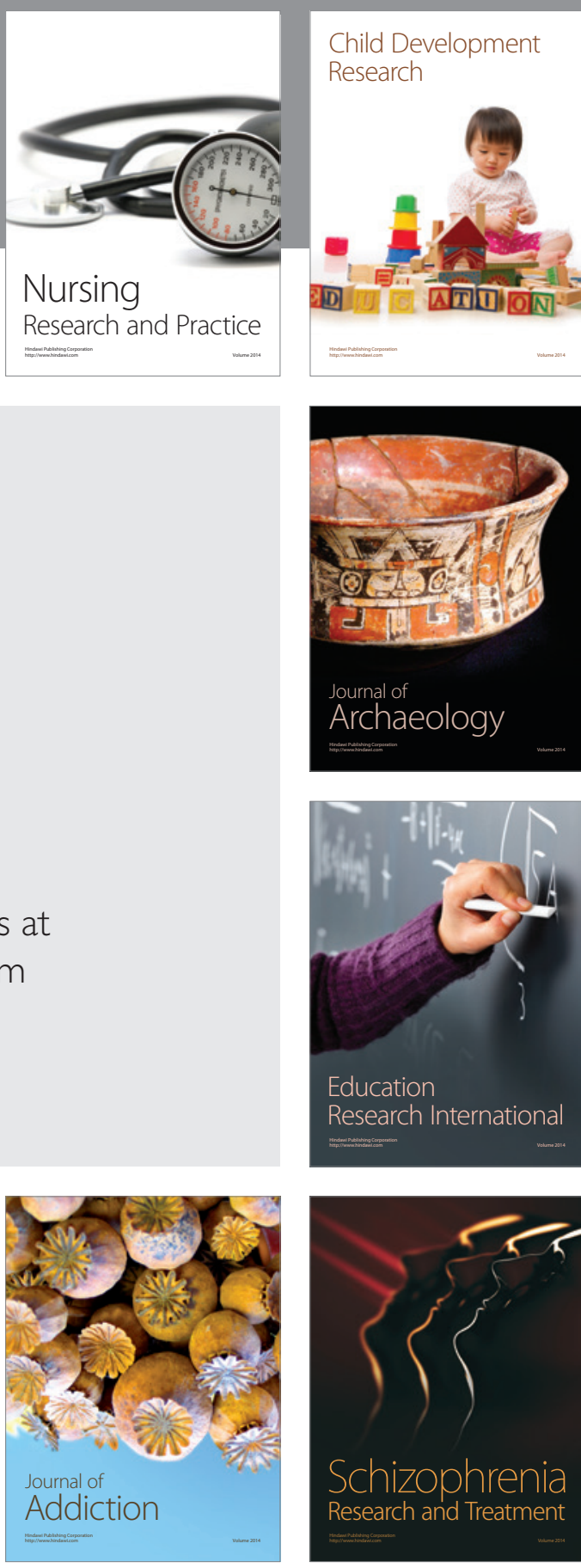

(D)
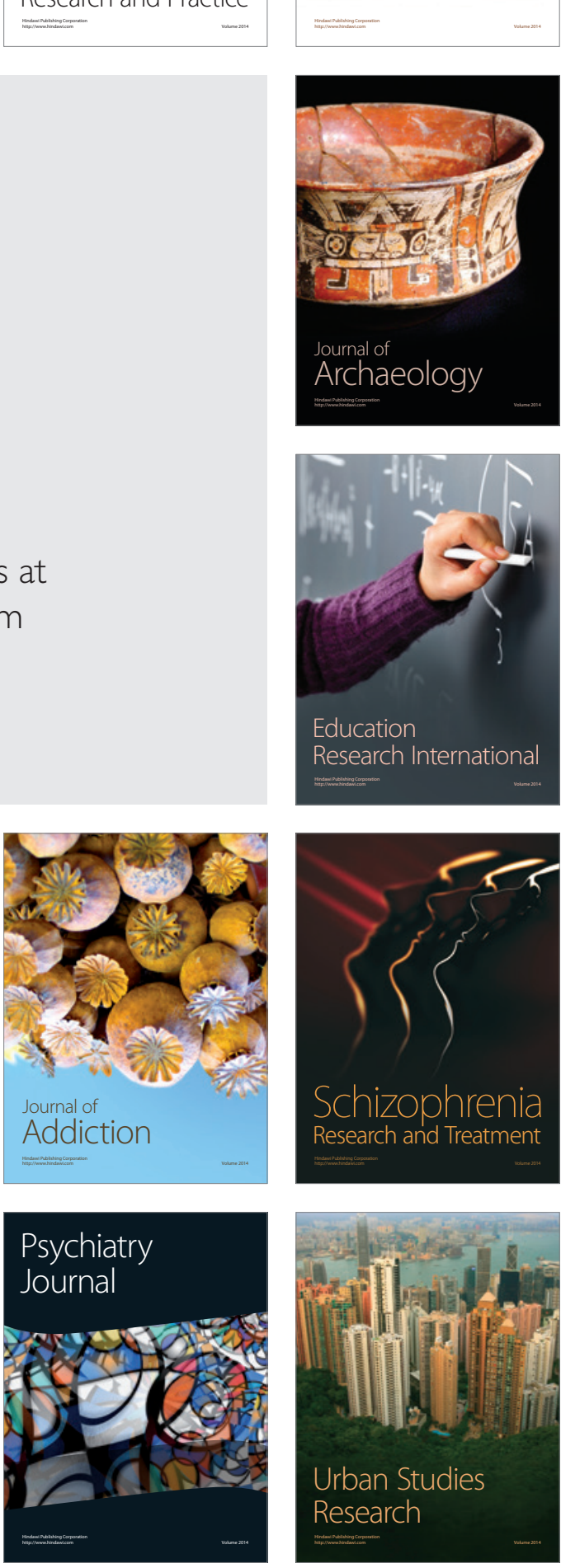